\title{
Application of Block-centered Finite Difference Formulation for Non-linear Finite Strain Consolidation
}

\author{
Dongseop Lee*, Chihun Sung**, Chulho Lee***, and Hangseok Choi ${ }^{* * * *}$
}

Received May 5, 2011/Revised March 26, 2013/Accepted November 10, 2013/Published Online June 20, 2014

\begin{abstract}
A one-dimensional block-centered finite-difference model has been developed to estimate the rate of non-linear finite strain consolidation. The governing equations including the hydrodynamic and constitutive equations are presented. The hypothesis of the uniqueness of the End-of-Primary (EOP) void ratio - effective stress relationship is adopted to calculate the primary consolidation settlement. The explicit block-centered finite difference formulations and boundary conditions are presented and discussed. The developed model was compared with a point-centered finite-difference program, ILLICON to show the efficiency of the blockcentered model. The block-center model provides an efficient tool to deal with interface boundaries and has advantageous ability to take into consideration the time-dependent loading, layered soil systems, and variable soil properties.
\end{abstract}

Keywords: soil consolidation, hydrodynamic equation, constitutive equation, block-centered finite-difference model

\section{Introduction}

The Terzaghi's theory of consolidation (1925) provides a rational basis for the computation of the rate and magnitude of settlement of fine grain soils. However, the utilization of the Terzaghi's theory in real problems is limited due to the simplified assumptions. Finite strain consolidation combined with the nonlinear behavior of clay deposits is essential in characterizing deformable behavior of very soft soils such as dredged fills. Duncan (1993), Olson (1998), and Terzaghi et al. (1996) emphasized the importance of considering the non-linear finite strain consolidation for the ultra-soft clay deposits. Mesri and Choi (1985a) developed the program ILLICON for modeling one-dimensional non-linear finite strain. The program ILLICON adopted the point-centered finite difference formulation to predict the settlement and progress of pore water pressure throughout the soil consolidation process. Mesri and Choi (1985b) proposed the hypothesis of the uniqueness of the End-Of-Primary (EOP) void ratio - effective stress relationship. The hypothesis becomes true when a unique EOP void ratio - effective stress relationship exists independently of the duration of the primary consolidation stage. In addition, Mesri and Choi (1985b) verified their EOP concept along with several long term monitoring programs. However, the uniqueness of the EOP void ratio is still under dispute and many research efforts on whether the EOP void ratio-effective stress relationship is unique or not is currently invested (Degago et al., 2009; Feng, 2010). In this study, this hypothesis is adopted in calculating the primary consolidation settlement.

In order to overcome the simplified assumptions in the Terzaghi's theory, various numerical methods have been used to take into account the complex behaviors of soil in the consolidation analyses. The most important advantages of the numerical model developed in this paper are the ability to incorporate the EOP void ratio - effective stress relationship during the soil consolidation that can be obtained using laboratory experiments and the ability to account for the variation of the other parameters such as the coefficients of consolidation (or permeability) and volumetric strain. In addition, the numerical model is able to take into account the stratified soil systems. Obtaining the analytical solutions considering the mentioned issues is hardly feasible due to the mathematical complication of the problems. The finite difference method is employed in this study to estimate the one-dimensional non-linear finite stain soil consolidation. Though this numerical technique has been used popularly in geotechnical analyses, the authors attempt to use the block-centered grid system instead of

*Member, Graduate Research Assistant, School of Civil, Environmental and Architectural Engineering, Korea University, Seoul 136-713, Korea (E-mail: steallady@korea.ac.kr)

${ }^{* *}$ Graduate Research Assistant, School of Civil, Environmental and Architectural Engineering, Korea University, Seoul 136-713, Korea (E-mail: hazlenut2@korea.ac.kr)

****esearch Engineer, Geotechnical Engineering Research Division, Korea Institute of Civil Engineering and Building Technology (KICT), Goyang 411712, Korea (E-mail: chlee@kict.re.kr)

****Member, Professor, School of Civil, Environmental, and Architectural Engineering, Korea University, Seoul 136-713, Korea (Corresponding Author, E-mail: hchoi2@korea.ac.kr) 
the point-centered grid system in this study. The block-centered grid system makes the finite difference formulation more versatile in dealing with the interface boundary condition between two different layers.

This paper presents a one-dimensional block-centered numerical model developed to simulate the process of the non-linear finite strain soil consolidation. The model incorporates the EOP void ratio effective stress relationship and the variation of the coefficients of consolidation and volumetric strain. The model is verified by comparing with the program ILLICON for a simple example problem.

\section{Governing Equations of One-Dimensional Non- linear Finite Strain Consolidation}

\subsection{Hydrodynamic Equation}

The derivation of the hydrodynamic equation begins with a differential element of soil with unit cross-sectional area which is in the progress of consolidation, as presented by Mesri and Choi (1985a \& b), Mesri and Lo (1989), and Lo (1991). The conservative law for the flow of pore water in the compressed element rules that the rate of volume change must be equal to the net flow rate, which results in the governing hydrodynamic equation as follows:

$$
\frac{1}{1+e} \frac{\partial e}{\partial t}=\frac{1}{\gamma_{w}} \frac{\partial}{\partial \xi}\left(k_{v} \frac{\partial u}{\partial \xi}\right)
$$

where $e$ is the current void ratio; $\xi$ is the height of the soil element during consolidation; $k_{v}$ is the coeffcient of permeability in the vertical direction, $u$ is the excess pore water pressure, and $\gamma_{w}$ is the unit weight of water.

The change in the coefficient of permeability during the consolidation can be obtained from the $e-\log k_{v}$ relationship as follows:

$$
\Delta \log k_{v}=\frac{\Delta e}{C_{k v}}
$$

where $C_{k v}$ is the ratio of a change in void ratio to a change in the logarithm of coefficient of permeability. If $C_{k v}$ can be assumed to be constant between the initial void ratio, $e_{0}$, and the EOP void ratio, $e_{p}$, the coefficient of permeability, $k_{v}$, at any void ratio, $e$, can be calculated as:

$$
k_{v}=k_{v 0} \exp \left[\frac{-2.3\left(e_{0}-e\right)}{C_{k v}}\right]
$$

where $k_{v 0}$ is the initial coefficient of permeability at the corresponding initial void ratio, $e_{0}$.

\subsection{Constitutive Equation}

The constitutive equation considered for the one-dimensional consolidation is:

$$
\frac{\partial e}{\partial t}=\left[\frac{\partial e}{\partial \sigma_{v}^{\prime}}\right]_{t} \frac{\partial \sigma_{v}^{\prime}}{\partial t}
$$

where $\partial e / \partial t$ is the change of the void ratio with time and governed by the permeability of soil as well as the boundary conditions, and $\left(\partial e / \partial \sigma_{v}^{\prime}\right)_{t}$ is the compressibility of the soil structure with effective stress. The rate of effective stress increase is:

$$
\frac{\partial \sigma_{v}^{\prime}}{\partial t}=\frac{\partial \sigma_{v}}{\partial t}-\frac{\partial u}{\partial t}
$$

Substituting Eq. (5) into Eq. (4), the constitutive equation becomes as:

$$
\frac{\partial e}{\partial t}=-\left[a_{v}\right]_{t}\left[\frac{\partial \sigma_{v}}{\partial t}-\frac{\partial u}{\partial t}\right]
$$

where $a_{v}$ is the slope of the EOP $e-\sigma_{v}^{\prime}$ relationship in both the compression and recompression ranges. Mesri and Choi (1985b) showed that the EOP $e-\sigma_{v}^{\prime}$ curves for soils are unique and are independent of the duration of the primary consolidation. Therefore, the result of the standard oedometer test on high quality samples can be used to estimate the in-situ settlement of the clay layers. The laboratory experimental data of the EOP $e-\sigma_{v}^{\prime}$ relationship is input into the current model as discrete points and, therefore, the change of value of $a_{v}$ is considered by approximating the $e-\sigma_{v}^{\prime}$ curve with linear segments.

Combining Eqs. (1) and (6), the governing equation for nonlinear finite strain soil consolidation to be solved in the model is as follows:

$$
-\frac{\left[a_{v}\right]_{t}}{1+e}\left[\frac{\partial \sigma_{v}}{\partial t}-\frac{\partial u}{\partial t}\right]=\frac{1}{\gamma_{w}} \frac{\partial}{\partial \xi}\left(k_{v} \frac{\partial u}{\partial \xi}\right)
$$

\section{Block-centered Finite Difference Formulations and Boundary Conditions}

\subsection{Block-centered Finite Difference Formulations}

An explicit finite difference approximation method is employed

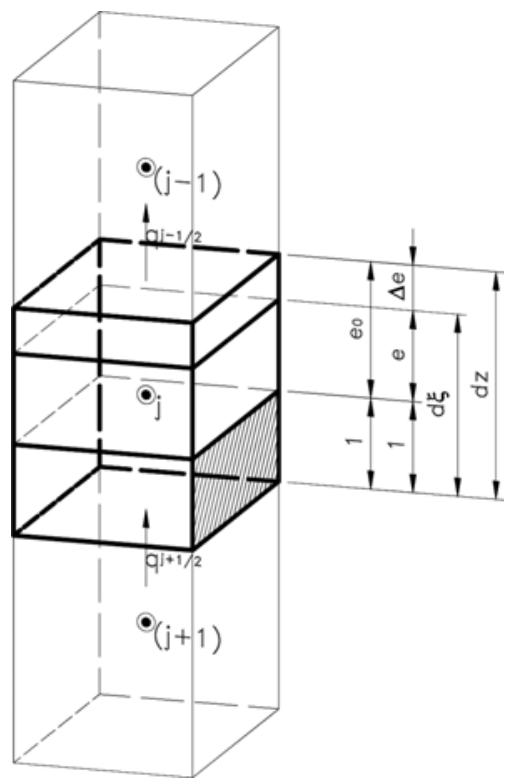

Fig. 1. Block-centered Model for Soil Consolidation 


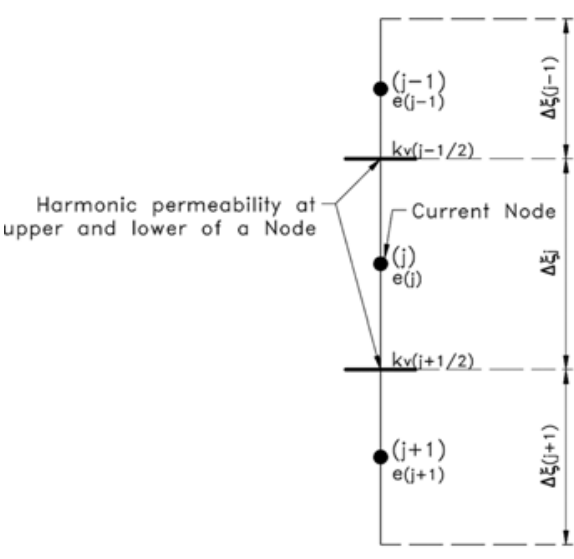

(a)

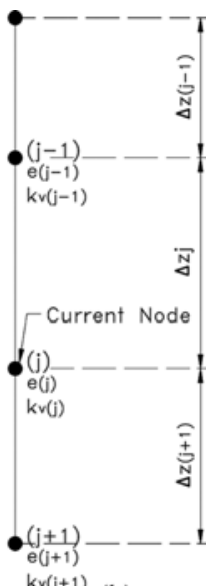

(b)
Fig. 2. (a) Grid Nodes and Inter-block Permeability for Block-centered Model, (b) Grid Nodes for Point-centered Model

to predict the non-linear finite strain soil consolidation. Fig. 1 shows the conceptual model for the block-centered finite-difference implementation. The finite difference formulations are built considering the soil block of node $j$ as shown in Fig. 1. The finite difference approximation is as follows:

$$
k_{v_{j+1 / 2}} \frac{h_{j}-h_{j+1}}{\frac{\left(\Delta \xi_{j+1}+\Delta \xi_{j}\right)}{2}}-k_{v_{j-1 / 2}} \frac{h_{j-1}-h_{j}}{\frac{\left(\Delta \xi_{j-1}+\Delta \xi_{j}\right)}{2}}=\frac{\Delta \xi_{j}}{1+e_{j}}\left[a_{v}\right]_{j}\left[\frac{\Delta \sigma_{v}}{\Delta t}-\frac{u_{j}^{t}-u_{j}^{t-\Delta t}}{\Delta t}\right]
$$

where $j$ indicates the layer number, $t$ means the current time step at which the values of pore water pressures in the soil layers are being calculated, and $(t-\Delta t)$ indicates the previous time step. The other quantities (i.e., $k_{v}, h, \Delta \xi, e$, and $a_{v}$ ) are obtained in the previous time step at $t-\Delta t$. The notations $k_{v_{j-1 / 2}}$ and $k_{v_{j+1 / 2}}$ are the inter-block permeability at the upper and lower interfaces of the current block $j$, respectively (see Fig. 2). The inter-block permeability is calculated by the subsequent equations. The other notations were described in the previous sections.

$$
\begin{aligned}
k_{v_{j-1 / 2}}= & \frac{k_{v_{j-1}} k_{v_{j}}\left(\Delta \xi_{j-1}+\Delta \xi_{j}\right)}{\Delta \xi_{j-1} k_{v_{j}}+\Delta \xi_{j} k_{v_{j-1}}} \\
= & \frac{k_{v_{j-1}} k_{v_{j}}\left(\left(\frac{1+e}{1+e_{0}}\right)_{j-1} \Delta z_{j-1}+\left(\frac{1+e}{1+e_{0}}\right) \Delta z_{j}\right)}{\left(\frac{1+e}{1+e_{0}}\right)_{j-1} \Delta z_{j-1} k_{v_{j}}+\left(\frac{1+e}{1+e_{0}}\right)_{j} \Delta z_{j} k_{v_{j-1}}} \\
k_{v_{j+1 / 2}} & \frac{k_{v_{j}} k_{v_{j+1}}\left(\Delta \xi_{j}+\Delta \xi_{j+1}\right)}{\Delta \xi_{j} k_{v_{j+1}}+\Delta \xi_{j+1} k_{v_{j}}} \\
= & \frac{k_{v_{j+1}} k_{v_{j}}\left(\left(\frac{1+e}{1+e_{0}}\right)_{j+1} \Delta z_{j+1}+\left(\frac{1+e}{1+e_{0}}\right) \Delta z_{j}\right)}{\left(\frac{1+e}{1+e_{0}}\right)_{j+1} \Delta z_{j+1} k_{v_{j}}+\left(\frac{1+e}{1+e_{0}}\right)_{j} \Delta z_{j} k_{v_{j+1}}}
\end{aligned}
$$

The value of pore water pressure at a node can be computed as:

$$
u_{j}^{t}=U P P E R(j)\left[u_{j-1}^{t-\Delta t}-u_{j}^{t-\Delta t}\right]+L O W E R(j)\left[u_{j+1}^{t-\Delta t}-u_{j}^{t-\Delta t}\right]+u_{j}^{t-\Delta t}+\Delta \sigma
$$

where

$$
\begin{gathered}
\operatorname{UPPER}(j)=k_{v_{j-1 / 2}} \frac{\left(1+e_{0}\right)_{j} \Delta t}{\gamma_{w}\left(a_{v}\right)_{j} \Delta z_{j}} \frac{2}{\left(\frac{1+e}{1+e_{0}}\right)_{j-1} \Delta z_{j-1}+\left(\frac{1+e}{1+e_{0}}\right)_{j} \Delta z_{j}} \\
\operatorname{LOWER}(j)=k_{v_{j+1 / 2}} \frac{\left(1+e_{0}\right)_{j} \Delta t}{\gamma_{w}\left(a_{v}\right)_{j} \Delta z_{j}} \frac{2}{\left(\frac{1+e}{1+e_{0}}\right)_{j+1} \Delta z_{j+1}+\left(\frac{1+e}{1+e_{0}}\right)_{j} \Delta z_{j}}
\end{gathered}
$$

The quantities $k_{v}, e$, and $a_{v}$ are updated at each time step based on the EOP $e-\sigma_{v}^{\prime}$ and $e-\log k_{v}$ relationships.

The dependent variable $(u)$ is explicitly obtained for a time step $(t+\Delta t)$ with the given initial and boundary conditions. This procedure is named a time marching procedure (or explicit method), which in some ways is simpler to handle than the implicit method. However, in the time marching implementation, the time step size $(\Delta t)$ should be dependent on the minimum grid size $\left(\Delta z_{\text {Min }}\right)$ and coefficient of consolidation $\left(c_{\mathrm{v}}\right)$ since the explicit method only satisfies a conditional stability. In a general onedimensional diffusion-type formulation (i.e., parabolic PDE), the time size step should satisfy the following convergence requirement.

$$
\Delta t<\frac{1}{2}\left(\frac{\left(\Delta Z_{\text {Min }}\right)^{2}}{c_{v}}\right)
$$

\subsection{Boundary Conditions}

The Neumann-type boundary condition is imposed on the boundary of the current block-centered grid system because the outmost nodes of the grid system are not located on the boundary of the considered domain but at the central points of the boundary blocks (Wang and Anderson, 1982; Thomas, 1995). The impervious boundary is simulated using the added imaginary blocks outside the considered domain. Each imaginary block is assigned a value of pore water pressure which is equal to the pore water pressure of the adjacent block within the considered domain. The drainage boundary on which the pore water pressure is equal to zero may bring about hindrance to the effort of using the block-centered model. Olson et al. (1974) introduced an alternative to simulate the drainage boundary for the pointcentered model by assigning the negative value of pore water pressure to the fictitious node at the boundary. The proposed scheme is employed in the current block-centered model as illustrated in Fig. 3(a). Chen et al. (2006) employed linear interpolation to compute the value of pore water pressure of the outmost node of the domain for the block-centered model from the values of pore water pressure of the predetermined boundary condition and the adjacent node as showed in Fig. 3(b). The linear interpolation technique is also an option to simulate boundary condition in the block-centered model.

The major advantage of the block-centered model is that the continuity of water flow is intrinsically incorporated into the finite 


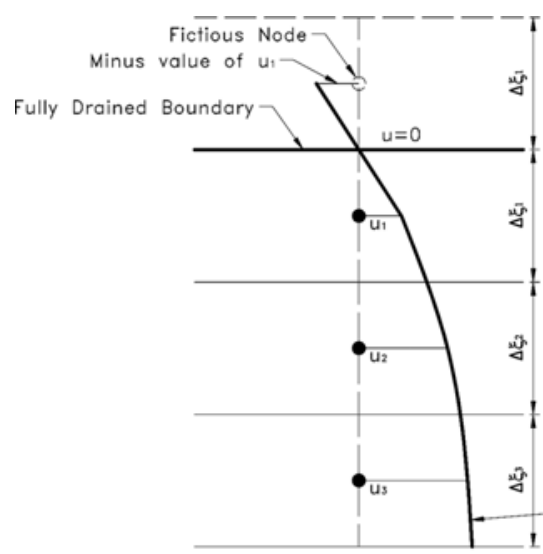

(a)

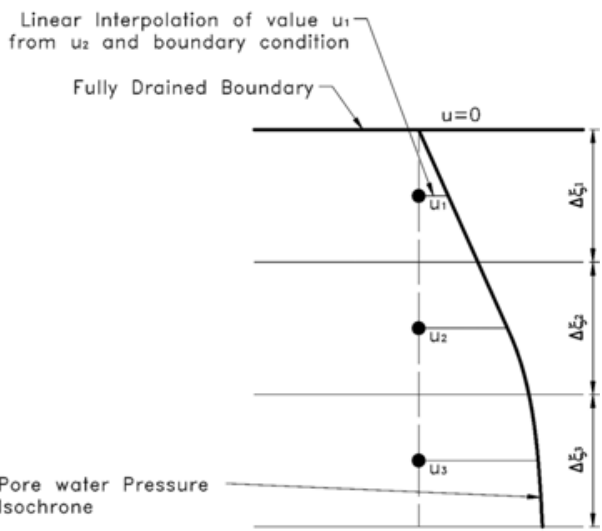

(b)

Fig. 3. (a) Drainage Boundary with Imaginary Block (Olson et al., 1974), (b) Linear Interpolation for Boundary Block (Chen et al., 2006)
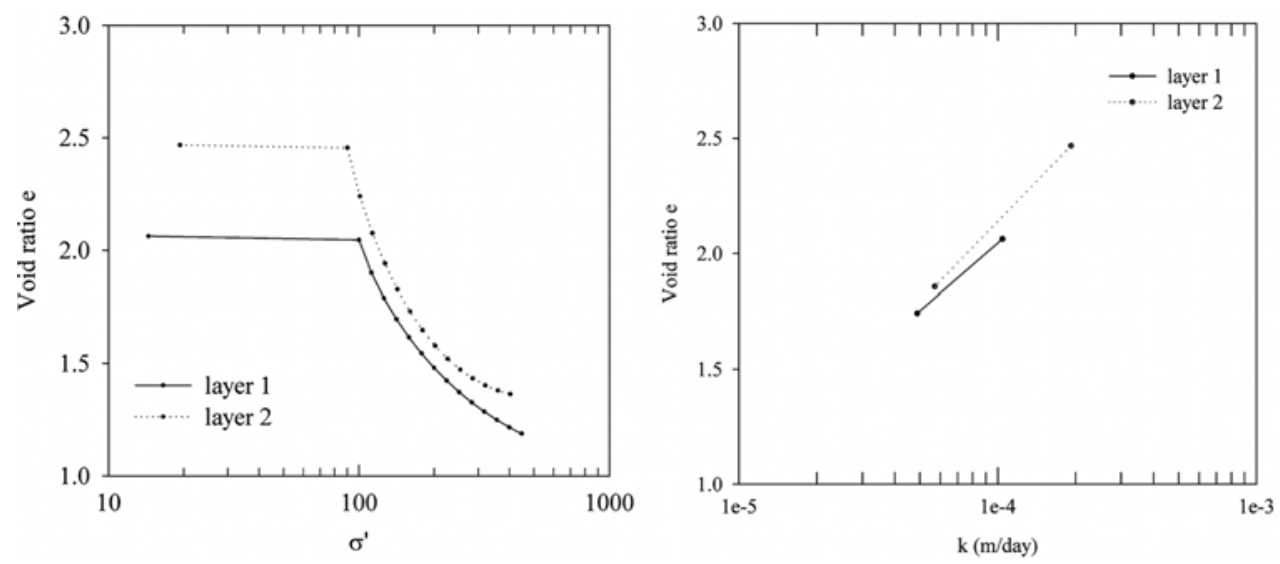

Fig. 4. $e-\sigma_{v}^{\prime}$ and $e-\log k_{v}$ Relationships for Clay Layers

difference formulations by adopting the harmonic average concept for the different hydraulic conductivities in two adjacent blocks. Therefore, it is not necessary to consider additionally the interface boundary condition between two adjacent blocks which have different hydraulic properties. Moreover, each block acts as an individual cell especially in the case that the hydraulic properties vary along with the change of effective stress in the consolidation problem. On the other hand, in the point-centered model, the different grid sizes of the layers and the nodes on the interface boundary which should have the properties belonging to both the upper and lower layers may result in complexities when building the code.

\section{Model Verification with Comparison of ILLICON}

An example problem of the one dimensional non-linear finite strain soil consolidation of the layered system is analyzed using the proposed block-centered model and the renowned program ILLICON (Mesri and Choi, 1985a \& b; Mesri and Lo, 1989; Lo, 1991). The program ILLICON was verified by many case studies (Mesri and Choi, 1985a \& b; Mesri and Lo, 1989; Lo, 1991). The finite strain and time-dependent loading are considered in this example. The nonlinear EOP $e-\sigma_{v}^{\prime}$ and linear $e-\log k_{v}$ relationships as the individual properties of each layer are taken into account (see Fig. 4). The soil formation comprises two clay layers with the thickness of $1 \mathrm{~m}$ for each layer. The boundary condition is selected as the two-way drainage. During the consolidation process, the void ratio and permeability are updated based on the nonlinear EOP $e-\sigma_{v}^{\prime}$ and linear $e-\log k_{v}$ relationships. The considered soil system is consolidated by a imaginary ramp loading as shown in Figs. 5, and 6. These figures also present the comparison of the results generated by the program ILLICON and the block-centered model proposed in this paper. The settlement is calculated by adopting the finite strain concept, which takes into account the EOP $e-\sigma_{v}^{\prime}$ relationship during the consolidation process and adjusts the layer thickness after each time step.

Along with the imaginary ramp loading, the evolution of pore water pressure predicted by the block-centered model agrees well with that by the program ILLICON except for the different maximum values of pore water pressure (see Fig. 5). In addition, the block-centered model yields the almost identical settlement to that by the program ILLICON (see Fig. 6). The minute difference in the calculated settlements may be attributed to the effect of secondary compression which was incorporated in the program ILLICON (Lo, 1991; Mesri et al., 1994). The blockcentered model does not incorporate the secondary compression 

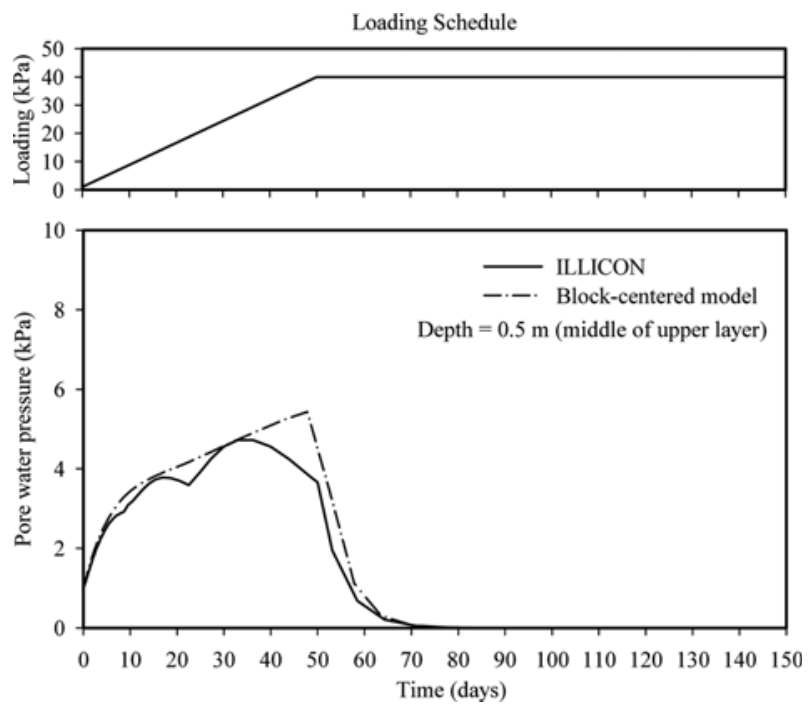

Fig. 5. Pore Water Pressure Evolution at Depth of $0.5 \mathrm{~m}$ by Ramp Loading
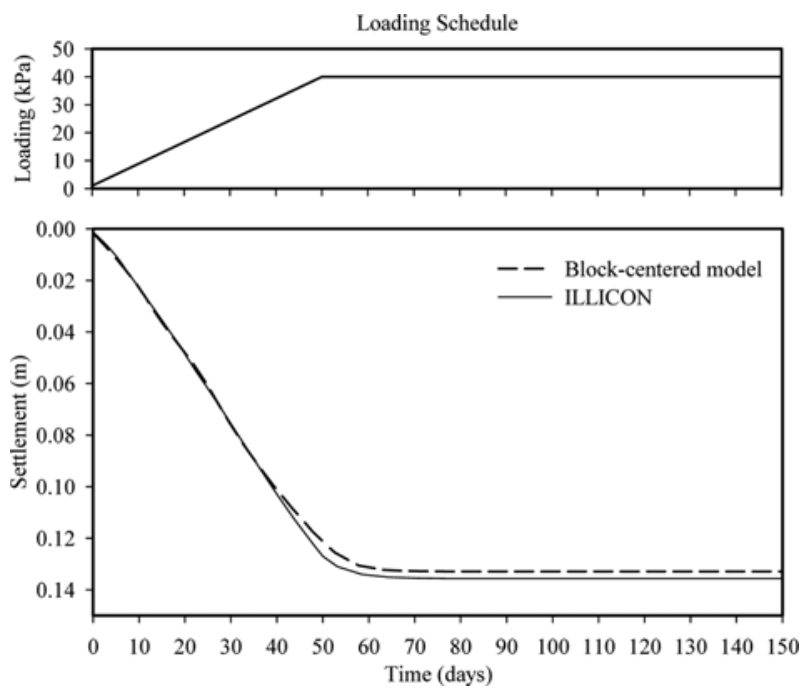

Fig. 6. Settlement by Ramp Loading

phenomenon. This lack may lead to the underestimation of settlement of a relatively thick clay deposit that requires a significant amount of time to finish consolidation.

\section{Conclusions}

The block-centered finite-difference model for one-dimensional non-linear finite strain soil consolidation has been developed in this paper. The hypothesis of the uniqueness of the End-OfPrimary (EOP) void ratio - effective stress relationship was adopted to calculate the primary consolidation settlement. The developed model can take into consideration the non-linear void ratio logarithm of coefficient of permeability relationship. The advantages of the block-centered model are the ability to consider the layered soil systems and time-dependent loading. The comparison of the block-centered finite-difference model and the renowned program
ILLICON shows the efficiency of the block-centered model. For future study, the model will be extended to incorporate the effect of secondary compression, horizontal drainage (i.e., three-dimensional problem), and the application of vertical drains.

\section{Acknowledgements}

This research was supported by a Korea University Grant (T1001611) and by a grant (12CTAPE02) from Construction \& Transportation Technology Advancement Research Program funded by Ministry of Land, Infrastructure and Transport (MOLIT) of Korea government.

\section{References}

Chen, Z., Huan, G., and Ma. Y. (2006). Computational methods for multiphase flows in porous media, Philadelphia, SIAM.

Degago, S. A., Grimstad, G., Jostad, H. P., and Nordal, S. (2009). "The non-uniqueness of the End-of-Primary (EOP) void ratio-effective stress relationship," Proceedings of the 17th International Conference on Soil Mechanics and Geotechnical Engineering, pp. 324-327.

Duncan, J. M. (1993). "Limitation of conventional analysis of consolidation settlement." Journal of geotechnical engineering, Vol. 119, No. 9, pp. 1333-1359.

Feng, T.-W. (2010). "Some observations on the oedometeric consolidation strain rate behaviors of saturated clay." Journal of GeoEngineering, Vol. 5, No. 1, pp. 1-7.

Lo, D. O. K. (1991). Soil improvement by vertical drains, PhD Thesis, Graduate College, University of Illinois at Urbana-Champaign, UrbanaChampaign, Ill., p. 292.

Mesri, G. and Choi, Y. K. (1985a). "Settlement analysis of embankments on soft clays." ASCE Journal of Geotechnical Engineering Division, Vol. 111, No. 4, pp. 441-464.

Mesri, G. and Choi, Y. K. (1985b). "The uniqueness of the End-Of-Primary (EOP) void ratio-effective stress relationship." Proceeding of 11th International Conference on Soil Mechanics and Foundation Engineering, San Francisco, California, pp. 587-590.

Mesri, G. and Lo, D. O. K. (1989). "Subsoil investigation: The weakest link in the analysis of test fills." The Art and Science of Geotechnical Engineering: At the Dawn of the Twenty-first Century, A Volume Honoring Ralph B. Peck, Prentice-Hall Inc., Englewood Cliffs, New Jersey, pp. 309-305.

Mesri, G., Lo, D. O. K., and Feng, T. W. (1994). "Settlement of embankments on soft clays." Geotechnical Special Publication 40, ASCE, Proc. Settlement'94, College Station, Texas, pp. 8-56.

Olson, R. E. (1998). "Settlement of embankments on soft clays." Journal of Geotechnical Engineering, Vol. 124, No. 8, pp 659-669.

Olson, R. E., Daniel, D. E., and Liu, T. K. (1974). "Finite difference analyses for sand drain problem." Proceedings of the Conference on Analysis and Design in Geotechnical Engineering, ASCE, Austin, Texas, Vol. 1, pp 85-110.

Terzaghi, K. (1925). Erdbaumechanik, Vienna, Franz Deuticke.

Terzaghi, K., Peck, B. P., and Mesri, G. (1996). Soil mechanics in engineering practice, $3^{\text {rd }}$ Ed., John Wiley \& Son.

Thomas, J. W. (1995). Numerical partial differential equations, finite difference methods, NewYork, Springer-Verlag.

Wang, H. F. and Anderson, M. P. (1982). Introduction to groundwater modeling, finite difference and finite element methods, W. H. Freeman, San Francisco. 